\title{
Effect of Grain Orientation and Cooling Rate on Stress Distribution in a Small-scale Silicon Ingot
}

\author{
SYLVAIN GOUTTEBROZE, ANTOINE AUTRUFFE, LARS MARTIN SANDVIK AAS, \\ MORTEN KILDEMO, and XIANG MA
}

\begin{abstract}
Small-scale solidification simulations were carried out in order to study the effect of the grain orientation and cooling rate on the stresses in mono- and bi-crystals. First, a 2D-axisymetric heat-transfer model of the global furnace is established to provide input to the sub-model. The sub-model takes into account only the crucible and silicon ingot. The flux histories are transferred from the global model. A finite element crystal plasticity model solves the mechanical deformation in the ingot. Ingots were grown in the small-scale Bridgman furnace with different pulling rates ranging from 0.2 to $50 \mathrm{~mm} / \mathrm{min}$. The results show the asymmetric effect of the crystal orientation and the stress build-up at the grain boundary due to different orientations. The change in pulling rate affects strongly the solidification front shape and the residual stresses. The 3D mechanical model illustrates also the limitations of the 2D-axisymmetric approach when silicon crystal anisotropy is taken into account.
\end{abstract}

DOI: $10.1007 / \mathrm{s} 40553-014-0018-5$

(C) The Minerals, Metals \& Materials Society and ASM International 2014

\section{INTRODUCTION}

THE solar cell industry is facing many challenges in its effort to continue increasing the cell efficiency with low market prices. The control of the defects in the current production process is essential to achieve that goal. The dominant technology, multicrystalline silicon cell, is facing specific problem related to the grain structure. Defects such as dislocations and certain grain boundaries diminish the material quality produced by directional crystallization. These defects are generated at high temperature during the ingot crystallization and enhanced by thermally induced deformations and mechanical interactions between the ingot and the crucible. Recent progresses in numerical simulation have allowed the analysis of complete furnace system including radiation, heat transfer, fluid flow, and segregation. ${ }^{[1,2]}$ The build-up of stresses and deformations during crystallization of silicon has been increasingly studied. ${ }^{[3-7]}$ However, these macroscopic models do not take into account the grains' orientations and their interaction. Some local study has been carried out. ${ }^{[8]}$ However, the complexity of industrial multicrystalline ingot does not allow for a full model simulation. Therefore, a small-scale furnace was selected to pursue the study of stresses and deformation during silicon

SYLVAIN GOUTTEBROZE and XIANG MA, Research Scientists, are with the SINTEF MK, Forskningsveien 1, 0340 Oslo, Norway. Contact e-mail: sylvain.gouttebroze@sintef.no ANTOINE AUTRUFFE, Ph.D. Student, is with the Department of Materials Science and Engineering, NTNU, A. Getz vei 2, 7491 Trondheim, Norway. LARS MARTIN SANDVIK AAS, Postdoctoral Researcher, and MORTEN KILDEMO, Professor, are with the Department of Physics, NTNU, Høgskoleringen 5, 7491 Trondheim, Norway.

Manuscript submitted October 30, 2013

Article published online May 22, 2014 crystallization. The purpose of the present work is to present a $2 \mathrm{D} / 3 \mathrm{D}$ model of a small-scale crystallization furnace and compare the model's prediction with the experiments of seeded directional solidification. The model is applied to analyze the effect of the cooling rate on the solidification and the grain orientation on the residual stresses.

\section{EXPERIMENTAL METHOD}

\section{A. Solidification Experiment}

Two types of small bi-crystal silicon ingots (40 and $60 \mathrm{~mm}$ in height with respective diameters of 40 and $32 \mathrm{~mm}$ ) were solidified using a Bridgman-type furnace. $\mathrm{Si}_{3} \mathrm{~N}_{4}$-coated alumina crucibles were used. Selected pulling rates were ranging from 0.2 to $50 \mathrm{~mm} / \mathrm{min}$. More details about the furnace and the solidification experiments method can be found in References 9, 10 . Seeded growth method was chosen in order to control the orientation of the two central grains and the orientation of the grain boundary plane and applied for the slowest pulling rate. The seeds were prepared out of a Czochralski monocrystalline ingot oriented in the $\langle 100\rangle$ direction. Two half cylinders axially oriented in the $\langle 110\rangle$ direction were prepared, and a controlled tilt misorientation was introduced between them, as explained in Figure 1; for more details, please refer to Reference 10.

The grain boundary plane was controlled, and the tilt angle was selected in order to grow the selected coincidence site lattice grain (CSL) boundaries. The seeds' manufacturing process is explained in Figure 1. In addition to the 5-deg misorientation presented in Reference 10 , the cutting angles $\alpha$ and $\beta$ were selected in order to build bi-seeds separated by $\Sigma 3\{111\}_{1,2}$ and 


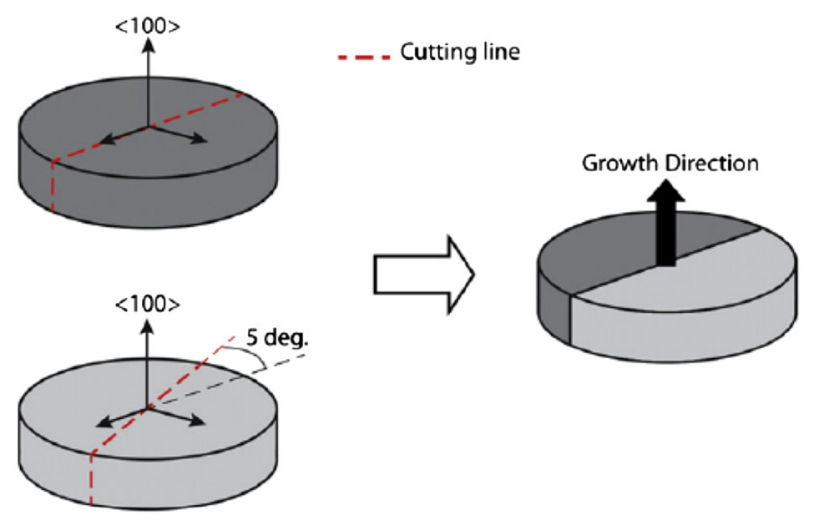

Fig. 1 - Seed preparation from $\mathrm{Cz}\left\langle\begin{array}{lll}1 & 0 & 0\end{array}\right.$ crystal. $^{[10]}$

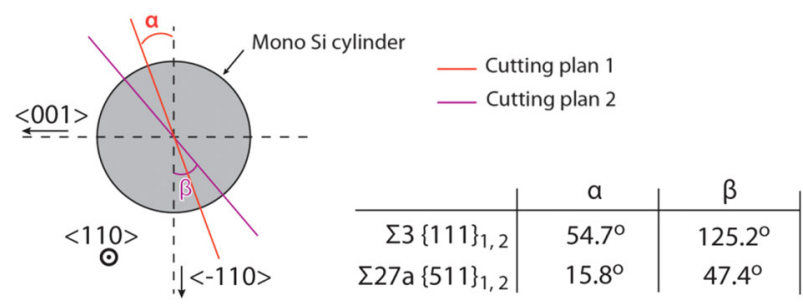

Fig. 2-Manufacturing of the $\Sigma 3\{111\}_{1,2}$ and $\Sigma 27$ a $\{511\}_{1,2}$ bi-crystal seeds from monocrystalline cylinders. Cutting angles are listed.

$\Sigma 27 \mathrm{a}\{511\}_{1,2}$ grain boundaries. Their nature, plane, and misorientation were confirmed by performing electron backscattered diffraction maps (EBSD) on horizontal cross sections (Figure 2).

\section{B. Strain Measurements}

Residual strains were measured in vertical cuts of the bi-crystal ingots, by means of near-infrared Mueller matrix imaging (MMI). As silicon is optically isotropic when the crystal is undeformed, the strain can be found by measuring the birefringence at every pixel in the image. The strain is proportional to the birefringence, which again is proportional to the retardance. Using $\mathrm{MMI}$, the retardance is found by analyzing the measured Mueller matrix image as explained in Reference 11. In particular, the Mueller matrix was decomposed to find the retardance using the polar decomposition method. ${ }^{[12]}$ Mueller matrix analysis also allows the inplane direction of the strain to be found as the orientation of the slow axis. The light source was a collimated 160-mW light emitting diode array with a center wavelength of $1300 \mathrm{~nm}$. The detector used was a Xeva indium-gallium-arsenide 2D array detector.

\section{MODEL DESCRIPTION}

The simulations have been carried out using Sisim, a heat-and mass-transfer model coupled with thermoelasto-viscoplastic model. Sisim has been developed at IFE and SINTEF based on previous work for aluminum industry. ${ }^{[6,7,13]}$ The evolving contact zones and air gaps between an ingot and the crucible are included in the thermal boundary conditions based on this full coupling. A surface-to-surface radiation model establishes the global energy balance in the furnace. The different domains are described using solid hexahedral eightnoded elements (4-nodes in 2D).

\section{A. Global and Local Model}

Based on a simplified design, the geometry of the small furnace was implemented in Gambit (distributed by ANSYS, www.ansys.com) using the crucible dimensions from Reference 9 (40-mm height and 40-mm diameter). In order to accurately model the crystallization of the ingot, a two-step approach has been used. In the first step, an axisymmetric mesh is built where all the parts are included (see Figure 3 ). The pulling procedure after the stabilization phase is modeled by a continuous displacement of the cup. The view factors are frequently updated to cope with the displacement of the cup (from 30 to 60 times depending on the pulling rate). The global furnace model is used to calibrate the volumetric power input in the resistive element and test sensitivity to material properties. However, for the mechanical calculations, a local model is preferred. The local model includes only the ingot and crucible domains. As presented in Figure 3(right), the external surface of the crucible is divided into 14 zones ( 9 on the lateral side, 3 on the bottom, 1 each on the top and on the interior). For each zone, the time-dependent average heat flux is computed from the 2D global furnace model.

\section{B. Constitutive Model}

The crystal plasticity model used in the simulation is described below (for more details, see Reference 14). It is assumed that the mechanism of plasticity in silicon is driven by slip-dominated flow (i.e., dislocation movements). The total strain tensor $\varepsilon$ is decomposed additively into a thermal part $\varepsilon_{\text {th }}$, an elastic part $\varepsilon_{\mathrm{e}}$, and a plastic part $\varepsilon_{\mathrm{p}}$, and the slip on each slip system (12 in $\mathrm{Si}$ crystal) is linked to the plastic gradients by

$$
\begin{gathered}
\varepsilon=\varepsilon_{\text {th }}+\varepsilon_{\mathrm{e}}+\varepsilon_{\mathrm{p}} \\
\dot{\varepsilon}^{\mathrm{p}}+\dot{\omega}^{\mathrm{p}}=\sum_{\alpha=1}^{12} \dot{\gamma}^{\alpha} s^{\alpha} \otimes n^{\alpha},
\end{gathered}
$$

where $s^{\alpha}$ and $n^{\alpha}$ are the slip direction and the normal to the slip plane, and $\dot{\varepsilon}^{\mathrm{p}}$ and $\dot{\omega}^{\mathrm{p}}$ are the plastic strain rate and the plastic spin, respectively. The resolved shear stress on each slip system is defined as a function of the Cauchy stress $\sigma$ which relates to the elastic strain via the elastic modulus tensor $K^{\mathrm{e}}$ :

$$
\begin{gathered}
\tau^{\alpha}=s^{\alpha} \sigma n^{\alpha} \\
\sigma=K^{\mathrm{e}}:\left(\varepsilon-\varepsilon^{\mathrm{th}}+\varepsilon^{\mathrm{p}}\right)
\end{gathered}
$$

The shearing rate on each slip system $\alpha$ is defined by a power-law relation: 


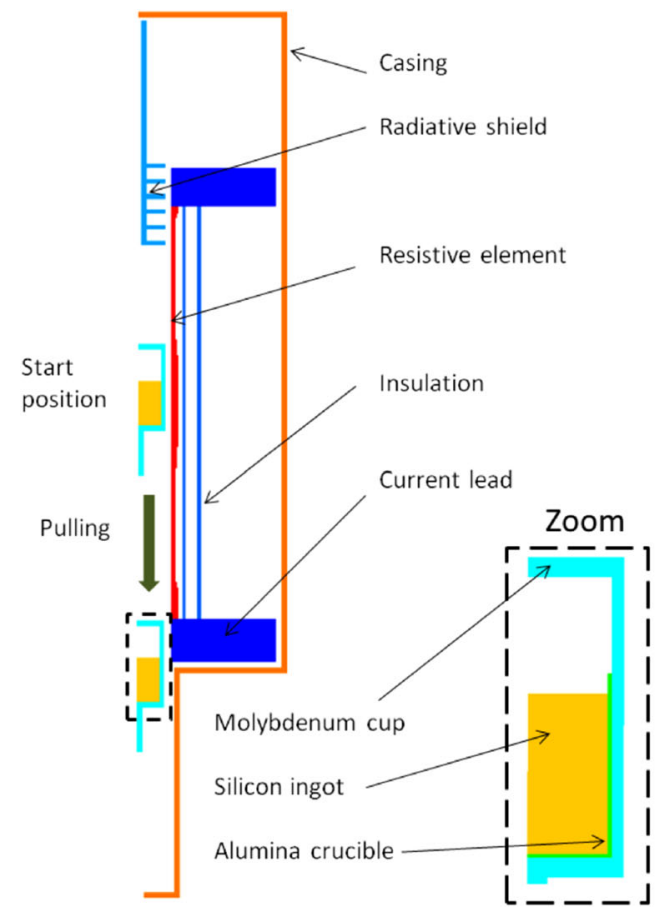

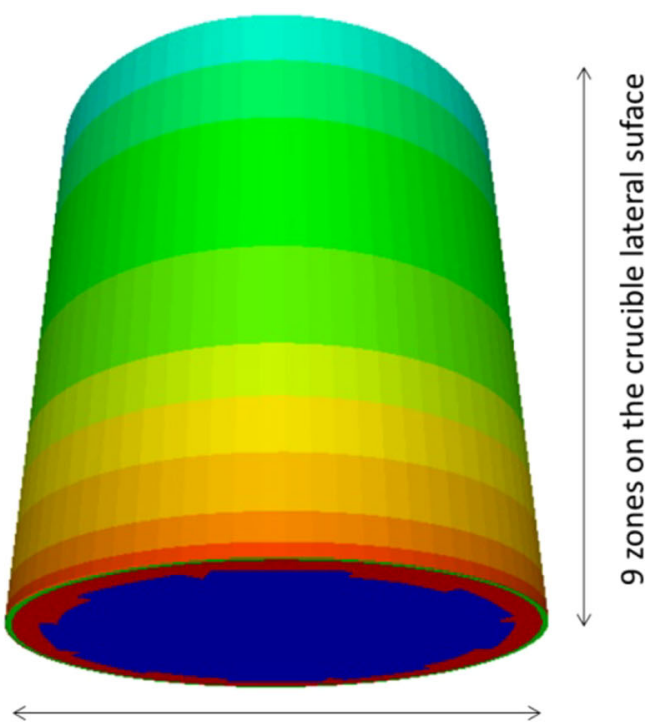

3 zones on the crucible bottom surface

Fig. 3- Global furnace model (left) and local model of crucible and ingot with heat flux discretization.

$$
\dot{\gamma}^{\alpha}=\rho^{\alpha} b v_{0} \exp \left(-\frac{Q}{k T}\right)\left\langle\frac{\tau_{\mathrm{eff}}^{\alpha}}{\tau_{0}}\right\rangle^{\frac{1}{m}} \operatorname{sgn}\left(\tau_{\mathrm{eff}}^{\alpha}\right),
$$

where $\rho$ is dislocation density, $b$ is the norm of the Burgers vector, $k$ is a Boltzmann constant, and $v_{0}, \tau_{0}, Q$, and $m$ are the material parameters (here $b=3.84 \times$ $10^{-10} \mathrm{~m} ; k=8.617 \times 10^{-5} \mathrm{meV} / \mathrm{K}, v_{0}=3.5 \times 10 \mathrm{~m} / \mathrm{s}$, $\tau_{0}=1 \mathrm{MPa}, Q=2.35 \mathrm{eV}$, and $\left.m=1\right)$. In addition,

$$
\begin{gathered}
\tau_{\mathrm{eff}}^{\alpha}=\tau^{\alpha}-\tau_{\text {int }}^{\alpha} \\
\tau_{\text {int }}^{\alpha}=\mu b \sum_{\beta=1}^{12} \sqrt{\alpha^{\alpha \beta} \rho^{\beta}}
\end{gathered}
$$

where $\mu=68.25 \mathrm{GPa}$. The evolution law for dislocation densities can be written as

$$
\dot{\rho}^{\alpha}=\left[P\left(\rho^{\alpha}\right)-A\left(\rho^{\alpha}\right)\right] \dot{\gamma}^{\alpha},
$$

where $P\left(\rho^{\alpha}\right)$ and $A\left(\rho^{\alpha}\right)$ represent the production rate and the annihilation rate of dislocations, respectively.

\section{NUMERICAL RESULTS AND DISCUSSION}

\section{A. Calibration of the Model}

The global furnace model has been calibrated using one temperature profile along the heater. This measurement was done with an empty furnace. As the temperature is controlled in the furnace, this temperature profile was expected to be very similar to the one obtained during standard crystallization run. The power input in the heater was adjusted to $16.3 \mathrm{MW} / \mathrm{m}^{3}$ to reach the same maximal temperature in the empty furnace. As presented in Figure 4(left), the temperature from the numerical model is very close to that of the experimental measurements. The model was also calibrated when the cup was included. The proximity of the cup from the heater changes the radiation heat transfer; therefore, the power input was adjusted to $13.1 \mathrm{MW} / \mathrm{m}^{3}$ in order to approximately reproduce the temperature measurements. Additional thermal measurement points during the crystallization process would be required for further validation of the model.

\section{B. Solidification and Thermal History}

After melting (partial melting of seeds is not modeled here) and holding at high temperature, the samples are cooled depending on the pulling rate. The macroscopic model considers silicon as an almost pure material leading to a temperature plateau at the melting point, while the material is solidifying as illustrated in Figure 5. The modeling results shown in Figure 6 indicate that for low pulling rates, solidification proceeds with an almost planar crystallization front, while higher cooling rate enhanced strongly the growth from the crucible surface. Only the first two cases will be used for comparison with experimental front shape and stress analysis. These results have been compared with experimental results as presented in Figure 5. The agreement between the model and experiment is satisfactory; it could be improved by a more thorough validation of the model.

\section{Strain and Stress Analysis}

For the analysis of the deformation during solidification, two configurations were defined as indicated in 


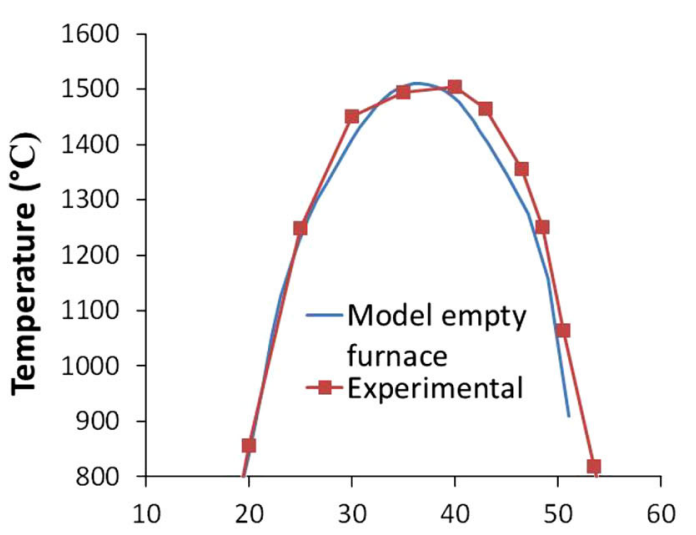

Height from bottom of the furnace $(\mathrm{mm})$

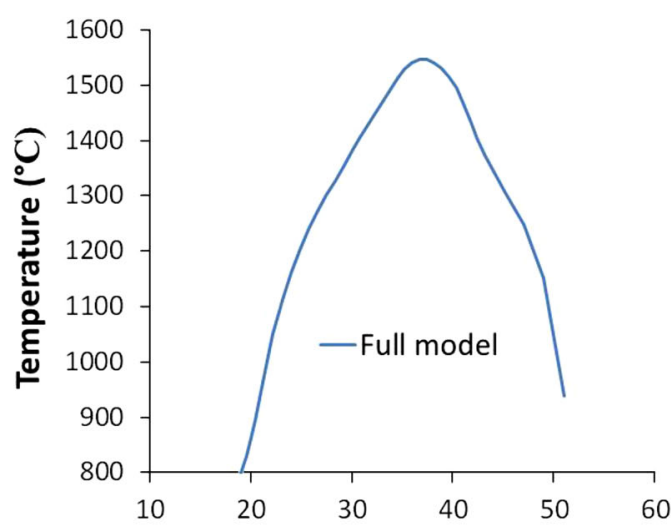

Height from bottom of the furnace $(\mathrm{mm})$

Fig. 4 - Comparison of experience and model temperature profile along the heater.
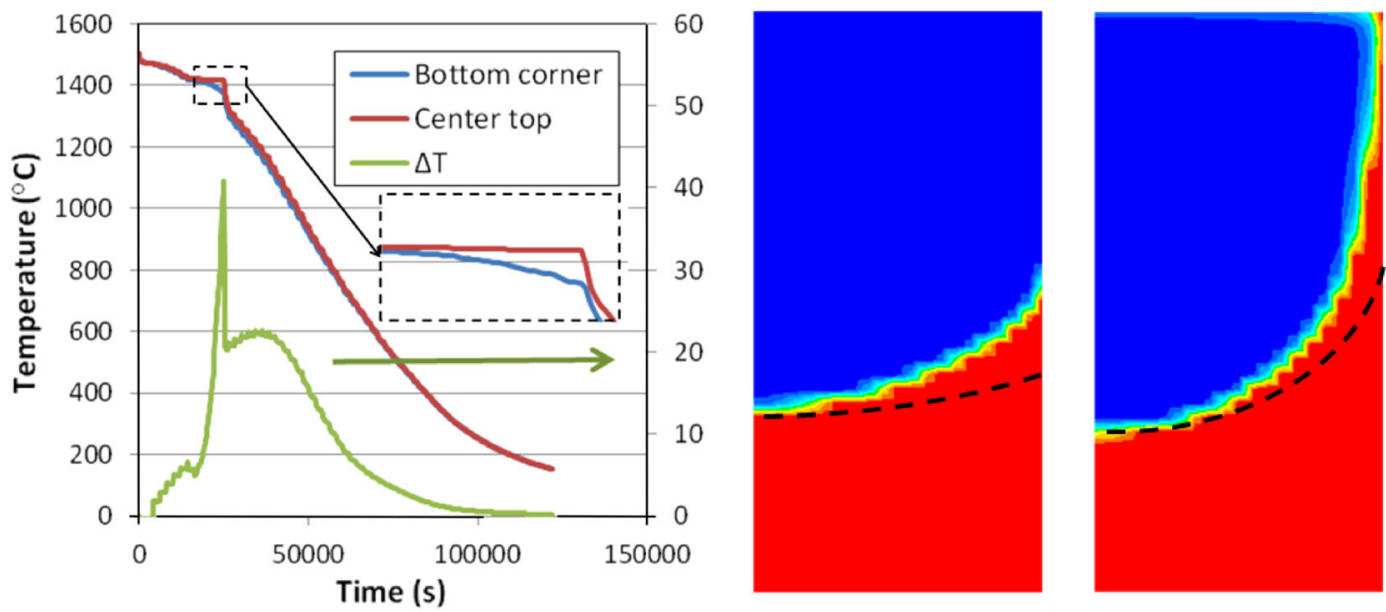

Fig. 5-Temperature history and gradient (left). Shapes of the solidification front for the same pulling rate (middle and right), respectively, 0.2 and $1 \mathrm{~mm} / \mathrm{min}$. The dash line indicates the approximate position of the solidification front measured experimentally. ${ }^{[15]}$
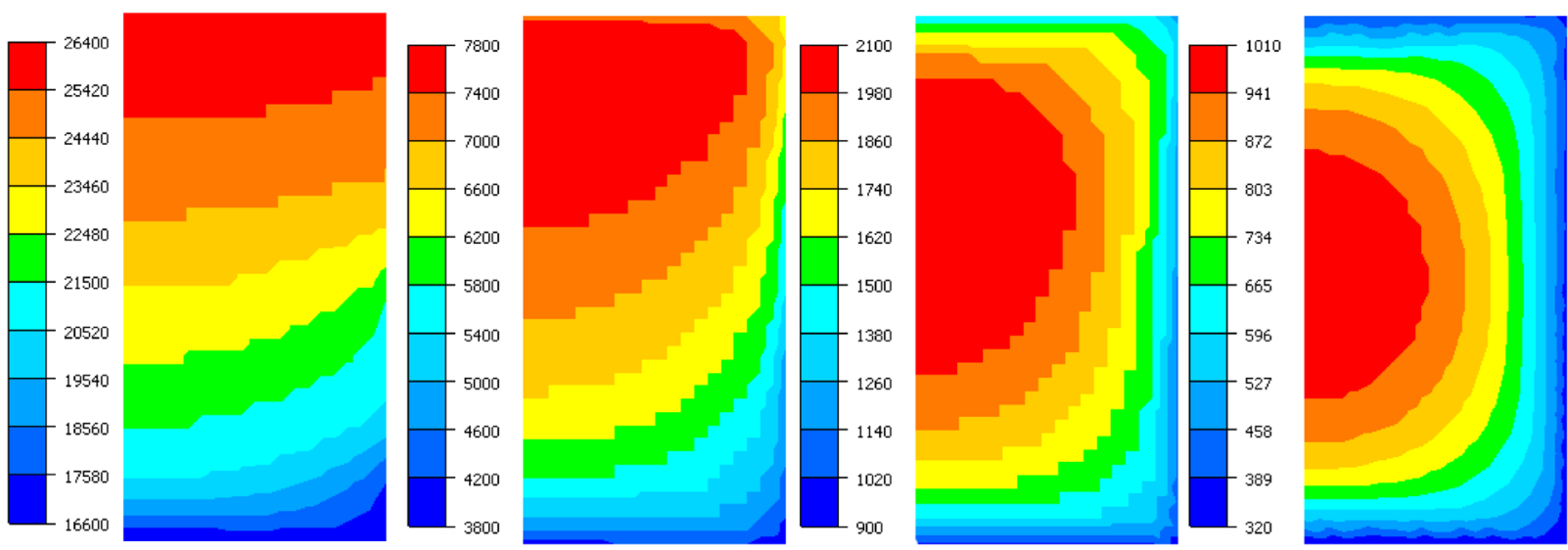

Fig. 6-Time of solidification for four different pulling rates from left to right: $0.2,1,8$, and $50 \mathrm{~mm} / \mathrm{min}$.

Figure 7. The orientations of the grain 1 and grain 2 were set into two configurations presented in the experimental method (5-deg rotations of grain 2 and sigma27 grain boundary).
As illustrated in Figure 8, the stress builds up at the coherency temperature (dark line in the left figure) specified in the model (just below melting point). The center is almost stress free for this solidification history 
and only in the contact with the crucible larger stresses can be reached. Therefore, only in these areas, the model will reveals effect of grain orientation as illustrated in Figure 8(a). Other contact models as well as thermal contraction of the crucible (currently assumed rigid)

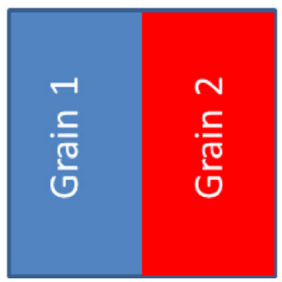

Case 1

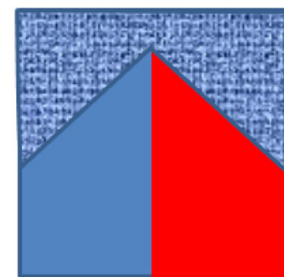

Case 2
Fig. 7-Configurations for stress analysis: Case 1 Bi-grain separated by a vertical plan with two grains; Case 2 Similar to case 1 but the end of the solidification is modeled as multicrystalline as observed in the experiments. should be applied to further study stress build-up at the grain boundary. When the pulling rate is increased, the maximum effective residual stress increases from 0.55 to $0.75 \mathrm{MPa}$, but larger stress values are reached locally during the solidification and cooling.

The stress field presented in Figure 9 is quite similar except those in the top corners, where no stress concentration is found due to the difference in the solidification front shape (see Figure 6). Figures 9(b) and (c) shows clearly the anisotropy of this stress field and illustrates why an axisymmetric approach is not appropriate when the grain orientation is taken into account. In all these simulations, the difference between the two grains configurations ( $5 \mathrm{deg}$ and $\Sigma 27$ ) is almost negligible using the current model.

\section{Comparison with Experimental Measurements}

The experimental measurements of the residual stresses using MMI technique are compared in Figure 10 with the

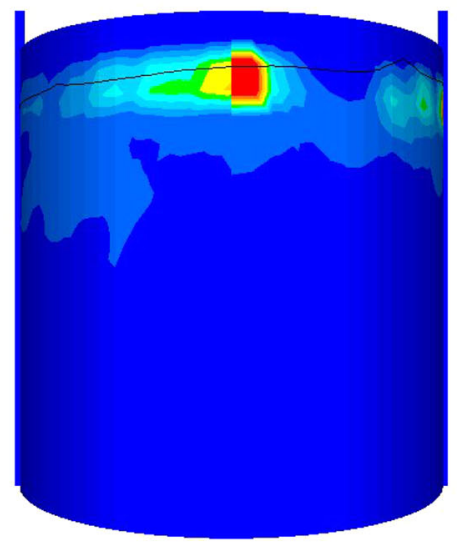

(a)

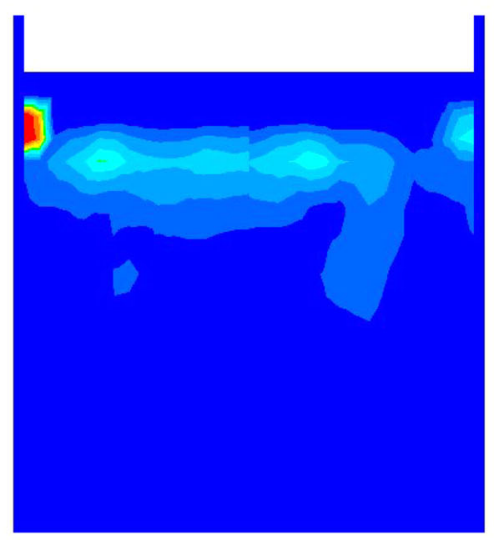

(b)

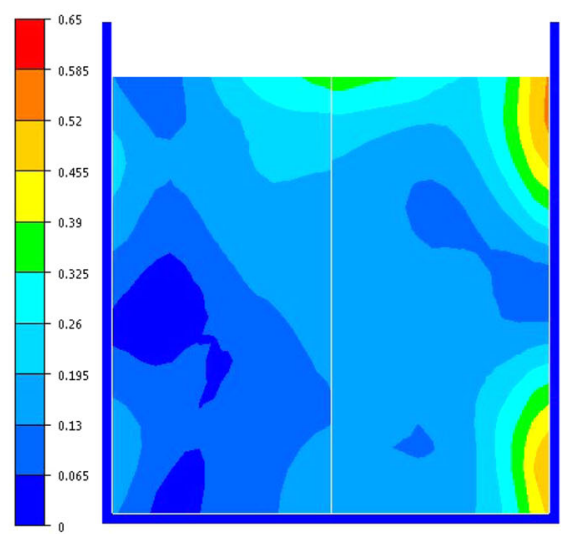

(c)

Fig. 8-Effective stresses on surface and central cross section for Case 1 with crystal plasticity for a 5-deg misorientations at 26,000 and $122,000 \mathrm{~s}$ (right). The scale is used for all figures. Maximum value is $0.65 \mathrm{MPa}$.

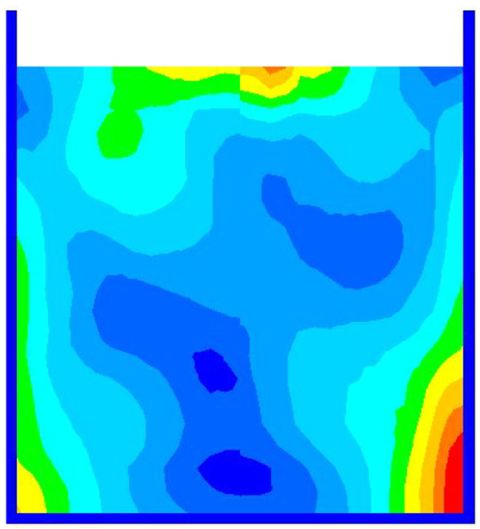

(a)

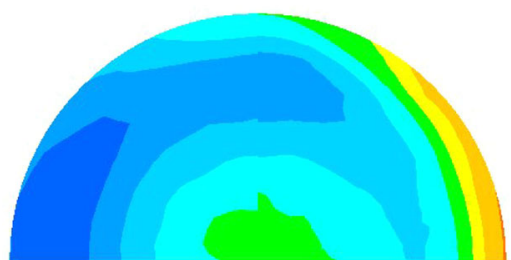

(b)

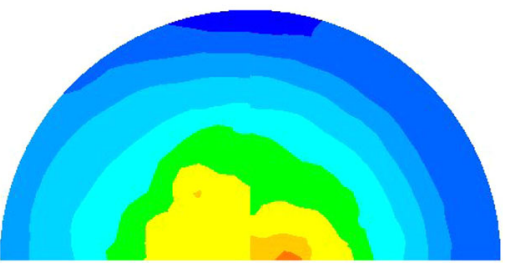

(c)

Fig. 9-Effect of pulling rate on effective residual stresses. Cross section for $1 \mathrm{~mm} / \mathrm{min}(a)$. Top views for 0.2 and $1 \mathrm{~mm} / \mathrm{min}$, resp. (b) and (c). The maximum value for figure (a) and (c) is $0.75 \mathrm{MPa}$, but only $0.65 \mathrm{MPa}$ for (b). 


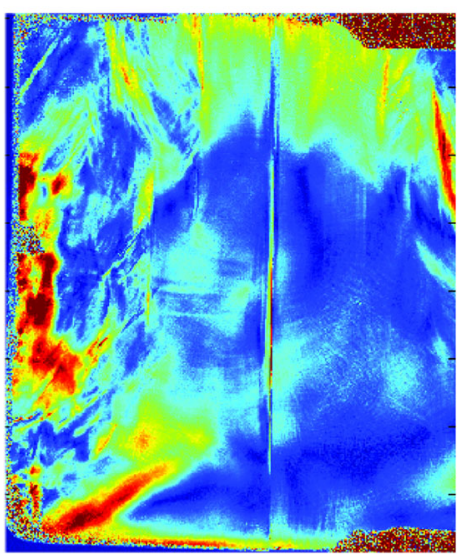

(a)

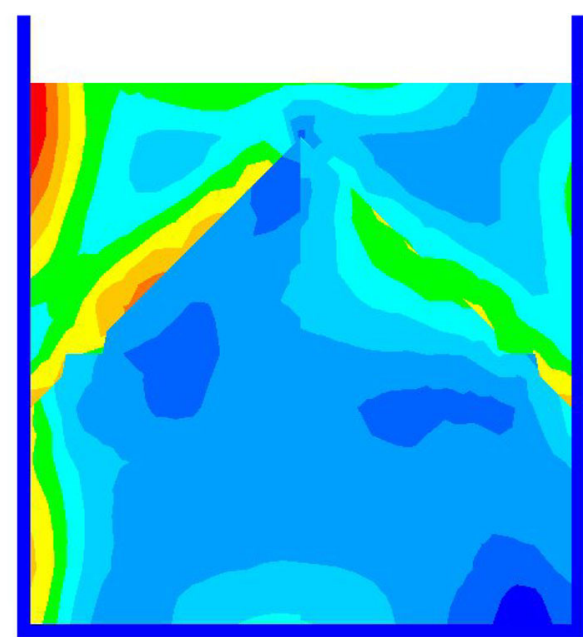

(b)

Fig. 10-Preliminary qualitative comparison of strain measurements made by MMI on $\Sigma 27$ a $\{511\}_{1,2}$ bi-crystal vertical cross section (left) with numerical effective residual stresses (right).

numerical model prediction. Three zones are present in the vertical section: (1) a central part with low stresses, (2) a higher-stress zone in the multicrystalline region formed at the end of the crystallization, and (3) dissymmetric high stress concentration at corners of the cross section. Each of these features is predicted with our numerical model. The relative amplitude of each is not entirely correct, but the pattern is captured by the model. Nevertheless, some aspects are not accounted for in the model, and therefore, the results are different. For example, the partial remelting of the seeds is not modeled so that additional stresses occur at the bottom especially in the center. The three material domains are predefined. While this assumption seems appropriate for the boundary between the two seeded grains, the transition between these grains and the more complex area at the end of the solidification is much smoother. The sharp stress transition as it appears in the model prediction is not present in the experimental measurements.

\section{CONCLUSIONS}

Directional bi-grain-seeded solidification experiments have been carried in a small-scale furnace with various cooling rates. The aim of this work is to model this experimental work in order to better understand the stress build-up due to grain orientation and boundary. For this objective, a 2D global model and a 3D local model have been established and partially validated by comparison of temperature and solidification measurements. The model illustrates the strong effect of the pulling rate on the front curvature. Two configurations - bi-grain (Case 1) and bi-grain plus a multicrystalline zone (Case 2) - have been simulated in the 3D thermomechanical model. The stress analysis shows that stresses arise mostly in the contact with the crucible, and while anisotropy has a significant effect, the model is not able to predict the effect of the grain boundary. The pulling rate increases the stress level and changes the stress field because of the higher front curvature. The comparison of Case 2 with MMI strain measurements shows a similar stress pattern except for the concentration at the boundary. Other contact models as well as thermal contraction of the crucible (currently assumed rigid) should be applied to further investigate stress build-up at the grain boundary. A finer mesh, especially at the grain boundary, might also reveal additional local stresses due to the grain misorientation.

\section{ACKNOWLEDGMENTS}

This work has been mainly performed within "The Norwegian Research Center for Solar Cell Technology" (Project Number 193829), a center for Environment-friendly Energy Research co-sponsored by the Norwegian Research Council and research and industry partners in Norway. The authors acknowledge the work done by S. Dumoulin and H.G. Fjær in implementing the crystal plasticity model.

\section{REFERENCES}

1. M.P. Bellmann, H. Dalaker, M. Syvertsen, S. Gouttebroze, and M. M'Hamdi: J. Cryst. Growth, 2013, vol. 362, pp. 38-41.

2. C. Reimann, M. Trempa, T. Jung, J. Friedrich, and G. Müller: $J$. Cryst. Growth, 2010, vol. 312, pp. 878-85.

3. X.J. Chen, S. Nakano, L.J. Liu, and K. Kakimoto: J. Cryst. Growth, 2008, vol. 310, pp. 4330-35.

4. X. Chen, S. Nakano, and K. Kakimoto: J. Cryst. Growth, 2010, vol. 312, pp. 3261-66.

5. M. M'Hamdi, E.A. Meese, H. Laux, and E.J. Øvrelid: Mater. Sci. Forum, 2006, vol. 508, pp. 597-602.

6. M. M'Hamdi, S. Gouttebroze, and H.G. Fjær: J. Cryst. Growth, 2011, vol. 318, pp. 269-74.

7. M. M'Hamdi, S. Gouttebroze, and H.G. Fjær: J. Cryst. Growth, 2013, vol. 362, pp. 83-87. 
8. I. Takahashi, N. Usami, K. Kutsukake, G. Stokkan, K. Morishita, and K. Nakajima: J. Cryst. Growth, 2010, vol. 312, pp. 897-901.

9. I. Brynjulfsen, L. Arnberg, and A. Autruffe: J. Cryst. Growth, 2012, vol. 361, pp. 206-11.

10. A. Autruffe, L. Vines, L. Arnberg, and M. Di Sabatino: J. Cryst. Growth, 2013, vol. 372, pp. 180-88.

11. L.M.S. Aas, P.G. Ellingsen, and M. Kildemo: Thin Solid Films, 2011, vol. 519 (9), pp. 2737-41
12. S.Y. Lu and R.A. Chipman: J. Opt. Soc. Am. A, 1996, vol. 13 (5), pp. $1106-13$

13. D. Mortensen: Metall. Mater. Trans. B, 1999, vol. 30B, pp. 11932.

14. J. Cochard, I. Yonenaga, S. Gouttebroze, M. M'Hamdi, and Z.L. Zhang: J. Appl. Phys., 2010, vol. 107, p. 033512.

15. A. Autruffe, M. Mhamdi, C. Modanese, L. Arnberg, and M. Di Sabatino Lundberg: unpublished research. 